\title{
Flow Field Optimization of SCR Reactor in Cement Plant
}

\author{
Zixiong Cao and Jian $\mathrm{Li}^{*}$ \\ Key Laboratory of Beijing on Regional Air Pollution Control, Beijing University of Technology, Beijing, 100124 China \\ ${ }^{*}$ Corresponding author
}

\begin{abstract}
In this paper the optimization for the flow field of a preliminary design of SCR device was studied of a cement plant production line with Fluent, which mainly aims to solve the problem of uneven distribution of velocity field by designing the gate leafs' and rectifiers' layout. To solve the problem of nonuniformity of velocity in SCR reactor, the angle of the wedge SCR reactor was adjusted, three non-equidistance "flat type" layout straight-arc-straight guide were designed by the method of similar geometry at the first bend and "inclined top type" rectifier was installed. Finally, the area weighted uniformity index value was more than $\mathbf{0 . 9 0 0 0}$ and the deviation angle was less than $10^{\circ}$, which provides ideas of the design and optimization method to the medium and small SCR reactors.
\end{abstract}

Keywords-numerical simulation; selective catalytic reduction (SCR); wedge; gate leafs; rectifiers

\section{INTRODUCTION}

With the constant improvement of China's environmental protection policy, the control of nitrogen oxides $\left(\mathrm{NO}_{\mathrm{x}}\right)$ emissions in our country is becoming increasingly strict. Selective catalytic reduction (SCR) is one of the most widely used de-nitration technologies, and it's widely used in the field of flue gas de-nitration[1-2]. However, with the emission standards become increasingly stringent, many existing SCR equipment is facing reconstruction.

The newly promulgated "Emission Standard of Air Pollutants for Cement Industry" (GB 4915-2013) on March 1, 2014, reduced the concentration of nitrogen oxides in cement industry from the previous $800 \mathrm{mg} / \mathrm{Nm}^{3}$ to $400 \mathrm{mg} / \mathrm{Nm}^{3}$, and in key areas the emission concentration limit is $320 \mathrm{mg} / \mathrm{Nm}^{3}$. While, in Beijing (DB $11 /$ 1054-2013), the concentration limit of nitrogen oxides emission is strictly limited to less than $200 \mathrm{mg} / \mathrm{Nm}^{3}$, the demand for de-nitrification projects of Beijing cement plants is more rigorous. In China, the SCR technology of cement kiln is immature, while, the rationality of SCR denitration process design has a direct impact of de-nitration efficiency on SCR. Using the numerical simulation technology can save the time, labor and resource for the transform of existing equipment. So the application of CFD simulation on equipment optimization has become an inevitable trend.

In this paper, Fluent software was used to reform and optimize SCR reactor in a certain cement plane in Beijing. The SCR reactor was designed according to the conditions and the flow field was optimized according to the gas velocity distribution, therefore, the design purpose was achieved, and methods could provide ideas for the design and reconstruction of SCR equipment.

\section{MOdEL Processing AND EVALUATION INDICATORS}

\section{A. SCR Structure Model and Mesh Processing}

1) Numerical Model

As is shown in Figure I, due to the limited space of the cement plant, the main of SCR reactor was designed as $4.8 \mathrm{~m}$ in length, $3.6 \mathrm{~m}$ in width and $11.3 \mathrm{~m}$ in height, and the inlet of the simulation area is a rectangular area of $3.6 \mathrm{~m}$ in length and $1 \mathrm{~m}$ in width.

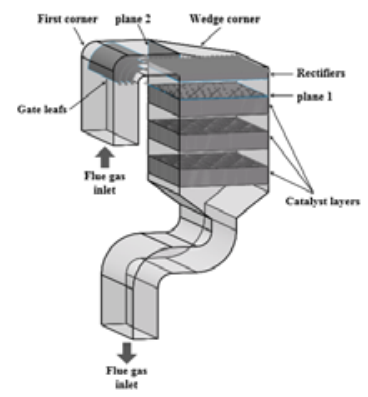

FIGURE I. SCHEMATIC OF CEMENT PLANT SCR FACILITY

2) Mesh Processing

As the geometric model was regular, the mesh was divided into hexahedral structured grid. At the area of installation of gate leafs and rectifier, the tetrahedral mesh was used to encryption, meanwhile the hexahedral mesh was encrypted in the area where the plane need to be detected (plane 1 and plane 2 ), and the mesh independent verification was carried out. Finally, the number of grids was 5 million, and the mesh model is shown in Figure II

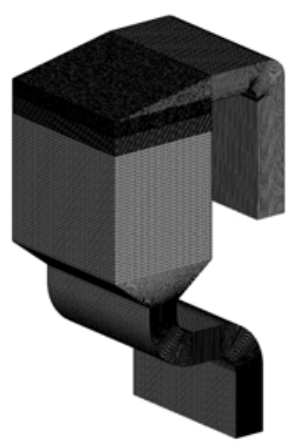

FIGURE II. THE MESH MODEL ADOPTED INTO SIMULATIONS 


\section{B. Numerical Simulation}

The simulation process was a three-dimensional flow analysis. According to the flow pattern of flue gas in a SCR system of a real industrial process, some simplifications and assumptions were made in the first step of investigations[3]: a) only one phase flows in the system, ignoring the effect of fly ash; b) the gas in the system is incompressible fluid; c) the system is adiabatic, ignoring the change of the temperature; d) gas flow in the system is steady state flow; e) ignoring the chemical reaction in the catalyst layers.

The gas in the SCR facility was controlled by the continuity equation, the energy equation, and the momentum equation[4], besides the standard $\mathrm{k}-\varepsilon$ turbulence model combining with the near wall function was adopted to capture the turbulence flow[5]. Using the SIMPLEC algorithm coupled with velocity and pressure, the low relaxation iterative factor method was used in iterative process[6]. The simulation process adopted the default upwind operation until the operation was converged. Therefore, the mesh was regulated by grid adaptively on the basis of velocity. Then the calculation accuracy was modified to second-order upwind until the calculation was converged.

The boundary condition of the entrance of flue gas was selected as velocity inlet, and the gas velocity was $14 \mathrm{~m} / \mathrm{s}$. The outlet was selected as the pressure outlet which was taken as 1 atm. The near wall area control used standard wall functions, and chose no slip boundary conditions.

\section{Evaluation Index}

Using the area-weighted uniformity index evaluates the homogeneity of the gas flow velocity distribution at the catalyst inlet plane (plane 1 in Figure I) and the wedge-shaped corner inlet plane (plane 2 in Figure II). Meanwhile, the standard deviation of the gas velocity distribution was used to help to evaluate the uniformity of the airflow distribution on plane 1 .

The area weighted uniformity index formula is as follow[7];

$$
\gamma=1-\frac{\sum_{i=1}^{n}\left[\left(\phi_{i}-\bar{\phi}\right) A_{i}\right]}{2|\bar{\phi}| \sum_{i=1}^{n} A_{i}}
$$

Where $\bar{\phi}$ is the average gas flow velocity on the detected plane; $A_{i}$ is the area of a single grid in a plane; $n$ is the total number of mesh in the plane. The area-weighted velocity uniformity index value is between 0 and 1 , with the increase of the value, the velocity distribution on a certain plane of the selected section would be better. The $\gamma$ value is an indicator of uniformity based on the area weights of different grid velocity[8], it's more suitable for the state of grids with different mesh sizes.

The formula of standard deviation is as follows:

$$
m=\sqrt{\frac{\sum_{i=1}^{n}\left(x_{i}-\bar{x}\right)^{2}}{n}}
$$

Where $x_{i}$ is the velocity of a single mesh in a certain plane; $\bar{x}$ is the average gas flow velocity on the detected plane; $n$ is the total number of mesh in the plane.

The aim of this project is that the area weighted average value of the catalyst inlet plane is greater than 0.9000 and the deflection angle of the airflow is less than $10^{\circ}$.

\section{Program Design AND SimUlation}

\section{A. Design of wedge SCR Reactor}

The slope of the wedge corner of the SCR reactor was studied. Wedge-shaped structure itself has the ability to guide the flow, if the slope was properly adjusted, it could not only save installation space and engineering costs, but also become a good flow guide element. Wang et al.[9], studied the design of the top of the wedge SCR reactor, and concluded that the uniformity of the flow field was better when the top wedge starting point and the inside right-angle corner inflection point were in the same vertical line. Based on the train of thought of Wang et al., the vertical position of the wedge end point was adjusted from the commonly used "full wedge" structure (the vertical wedge height $\Delta \mathrm{H}=800 \mathrm{~mm}$, which was on the same horizontal line as the inflection point of the internal right-angle corner) to transitional "trapezoidal wedge-shaped top structure" $(\Delta H=600 \mathrm{~mm})$ a series of chamfering vertical height scheme was designed, aiming to find the best design of the top wedge SCR structure that a better gas-velocity distribution at the catalyst inlet plane could be given without baffle correction.

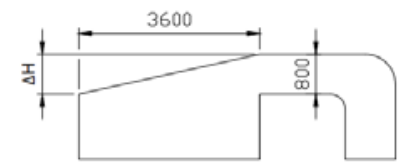

FIGURE III. THE DESIGN METHOD OF TOP CHAMFER OF SCR EQUIPMENT

The vertical height value $(\Delta H)$ of SCR reactor wedgeshaped top chamfering in this series was: $\Delta H=600 \mathrm{~mm}, 610 \mathrm{~mm}$, $620 \mathrm{~mm}, 630 \mathrm{~mm}, 640 \mathrm{~mm}, 650 \mathrm{~mm}, 660 \mathrm{~mm}, 680 \mathrm{~mm}, 700 \mathrm{~mm}$, $720 \mathrm{~mm}, 740 \mathrm{~mm}, 760 \mathrm{~mm}, 780 \mathrm{~mm}, 800 \mathrm{~mm}$. The standard deviation and the area weighted uniformity index $\gamma$ value of the gas flow velocity at the inlet plane of catalyst layer (plane 1) are shown in Figure IV.

According to Figure IV, when $\Delta \mathrm{H}$ value was $640 \mathrm{~mm}$ and $650 \mathrm{~mm}$, the $\gamma$ value of catalyst inlet plane was significantly higher than other programs in this series. When $\Delta H=650 \mathrm{~mm}$, the standard deviation of gas velocity distribution of plane 1 was obviously lower than $\Delta \mathrm{H}=640 \mathrm{~mm}$, and the standard deviation of $\Delta \mathrm{H}=650 \mathrm{~mm}$ was the smallest in the whole series, so it could be concluded that the gas velocity was even better. Taken together, the choice of wedge height of $\Delta H=650 \mathrm{~mm}$ was the most suitable program among this series. 


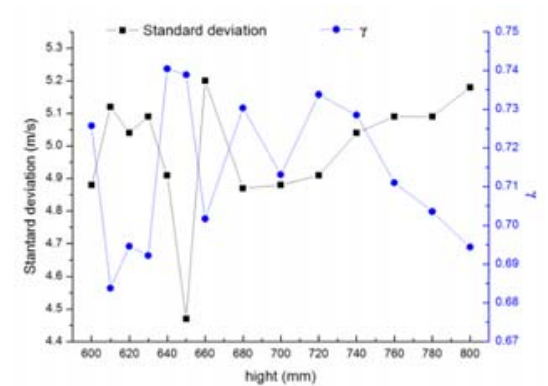

FIGURE IV. SIMULATION RESULTS OF SERIES ONE
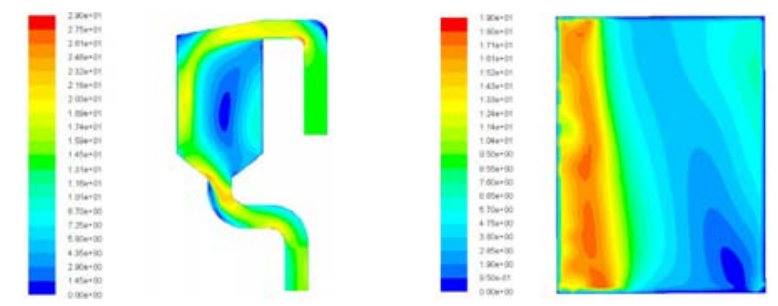

FIGURE V. VELOCITY CONTOURS OF AXIAL SYMMETRY PLANE (LEFT) AND CATALYST INLET PLANE (RIGHT) OF $\Delta \mathrm{H}=$ $650 \mathrm{MM}$

As could be seen from Figure V, the velocity field became very disordered after flue gas had passed through the corners due to the absence of the diversion device. This unevenness mainly manifested in: a) there was a triangular low-speed zone at the outside of right-angled bend; b) after the corners, the inside of the flue was low speed area, the outside was high speed area, the speed difference was very large; c) at the second corner, a low speed zone appeared on both the inner and outer sides due to the large size variations.

The main reason for this non-uniformity was that when the flue gas flowed through the bend, its flow line would diverge, while due to inertia, the fluid would be forced to the outside, resulting in an increasing of pressure of the fluid outside the elbow. According to the Bernoulli equation, the speed of the fluid outside the curve was small, and then the pressure decreased gradually until the curve terminates after some point in the middle of the curve, the distribution of gas velocity and pressure inside the curve was opposite to the outside of the curve. This phenomenon occurred mainly in the first corner, therefore, to improve the velocity field of the entire flue, the first priority was to mitigate the effect of the first corner on the velocity field.

\section{B. Design of Baffles at the First Bend}

Baffles installation was the main method to optimize the airflow distribution inside the SCR reactor. If the arrangement of baffles was reasonable could not only make the flow field in the reactor more uniform, but also reduce the system pressure loss. Xu Yan et al.[10] studied four kinds of baffles and found that the combination of straight baffles and curved baffles had a better effect on flow field optimization. In this paper, the straight-arc-straight baffle was used to optimize the flow field at the first curve and the best baffle plate number and arrangement were determined by equidistant layout. Then via changing the distance between each two deflectors, a better design to make gas flow rate more uniform was explored.
The number of baffles was chosen as two, three and four, and there were "flat" type and "gradient" type of baffle layout methods. Plan N represented no guide plates; plan A was "flat" layout of two straight-arc-straight baffles; Plan B was "gradient" layout of two straight-arc- straight baffles; Plan A' was "flat" layout of three straight-arc-straight baffles; Plan B' was "gradient" layout of two straight-arc- straight baffles; Plan A" was "flat" layout of four straight-arc-straight baffles; Plan B" was "gradient" layout of four straight-arc-straight baffles. Specific installation and simulation results were shown in Figure VI and Table I below.

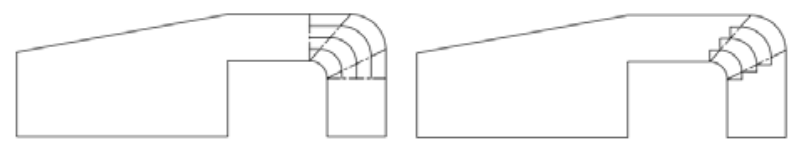

FIGURE VI. "FLAT" (LEFT), "GRADIENT" (RIGHT) GUIDE PLATE LAYOUT (TAKE THREE BAFFLES FOR EXAMPLE)

TABLE I. SIMULATION RESULTS OF SERIES 2

\begin{tabular}{|c|c|c|c|}
\hline Type & $\begin{array}{c}\text { Plane 1mean } \\
\text { velocity }(\mathrm{m} / \mathrm{s})\end{array}$ & $\gamma_{1}$ & $\gamma_{2}$ \\
\hline N & 8.94 & 0.7389 & 0.9596 \\
\hline A & 10.08 & 0.7542 & 0.9651 \\
\hline B & 9.41 & 0.7475 & 0.9625 \\
\hline A' & 10.01 & 0.7689 & 0.9689 \\
\hline B' & 10.01 & 0.7537 & 0.9684 \\
\hline A" & 9.21 & 0.7467 & 0.9668 \\
\hline B" & 7.65 & 0.7145 & 0.9780 \\
\hline
\end{tabular}

As could be seen from Table I, the optimization effect on the overall flow, the "flat" layout method of the baffles on field was generally better than that of the "gradient" layout of the baffles, placing the baffles at the first bend had a significant effect on the airflow optimization of plane 1. But not the more number of baffles set, the better optimization effect of the flow field had. That was due to the increasing number of baffles made pressure loss value get higher, and the flow directions of the airflow were more difficult to be changed. Considering the values of $\gamma_{1}$ and $\gamma_{2}$, the flow field optimization effect of plan A' was thought as the best. Therefore, the number of baffles was selected to be three and the baffles were arranged in a "flat" form.

According to the airflow distribution without diversion measures, it can be known that the airflow velocity distribution at the starting point of the baffle arranged in the Plan A' was large near the inner side of the bend, and the gas velocity outside was small. To further improve the airflow optimization effect of the baffles at the right-angle bend on the SCR equipment, the spacing of the baffles in Plan A' could be adjusted, to achieve the purpose of uniform segmentation of the cross-section gas flow rate.

The layout of Plan $\mathrm{C}$ was as follows: the horizontal spacing (mm) of the starting point of the baffle was 175: 225: 262.5: 337.5 ; the vertical spacing $(\mathrm{mm})$ of the ending point was 140 : 180: 210: 270 ; the flux $\left(\mathrm{m}^{3} / \mathrm{s}\right)$ ratio was 16.20: 16.88: 16.57: 17.13. The simulation results of the scheme $\mathrm{C}$ were as follows: the area weighted index value of velocity distribution $\left(\gamma_{1}\right)$ of plane 1 was 0.7862 ; the area weighted index value of velocity 
distribution $\left(\gamma_{2}\right)$ of plane 2 was 0.9714. Compared with Plan A' $\left(\gamma_{1}=0.7689, \gamma_{2}=0.9689\right)$, there was a significant improvement.
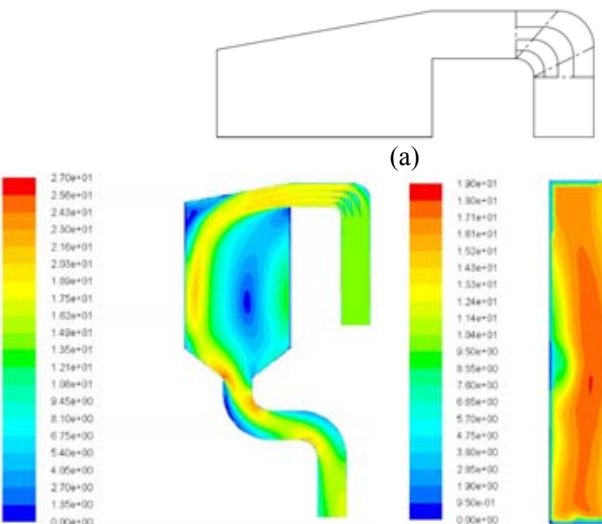

(a)

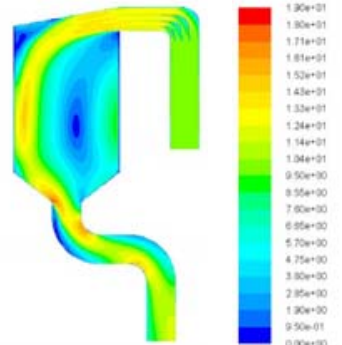

(b)

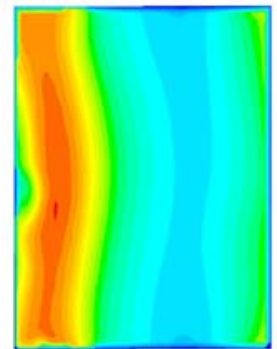

(c)

FIGURE VII. THE DESIGN METHOD OF PLAN C (A) AND ITS VELOCITY CONTOURS OF AXIAL SYMMETRY PLANE B) AND CATALYST INLET SECTION (C)

From Figure VII, compared with the air flow distribution without optimization method, the low velocity triangle at the outer side of the first bend disappeared and the air velocity distribution was more uniform after curve. The area of high velocity inside the SCR main body was reduced and moved inwards, and the low velocity swirl zone was reduced. The gas flow distribution at plane 1 was obviously improved.

\section{Determination of Rectifier Grid Layout Program}

The "ramp top" rectifier grid was installed below the wedge-shaped top structure. The top point of the grid connection was collinear with the start of the curve and the end of the wedge, the bottom of the grid was at the same level, the vertical distance between the bottom of the rectifier grid and the inflection point inside the right angle was $300 \mathrm{~mm}$. 35 plates were consisted in the rectifier, the spacing between the plates was $100 \mathrm{~mm}$, the length of the plate increases one by one, the increasing value was $4.17 \mathrm{~mm}$. The specific installation form and flow field simulation results of the rectifier were shown in Figure VIII.

As could be seen from Figure VIII (b), the uniformity of velocity field improved obviously after the installation of the rectifier, and the eddy field inside the SCR device disappeared. From Figure VIII (c), most of the regional gas velocity values was between $4 \mathrm{~m} / \mathrm{s}$ and $5 \mathrm{~m} / \mathrm{s}$, in the range of theoretical gas velocity value. The standard deviation of gas velocity distribution of Plane1 was $0.79 \mathrm{~m} / \mathrm{s}$, the fluctuation of gas flow was small, and the area weighted uniformity index $(\gamma)$ value was 0.9226 , the maximum airflow deflection angle was under $8^{\circ}$ which all meeting the design requirements.

\section{CONCLUSION}

Through the design of the wedge SCR reactor shape, the purpose of using the wedge structure to guide the flow field was achieved. Thereby, the necessity of installing the baffle inside the wedge-shaped top member and the construction difficulty were reduced.
By installing the deflector and the rectification grid, the uniformity of the airflow distribution was improved at the catalyst inlet plane. The uniformity index value increased from 0.7389 that without any flow field optimization measures to 0.9226 , the maximum airflow deflection angle was about $8^{\circ}$, reached the engineering design goal and these methods could provide a reference for the similar design.

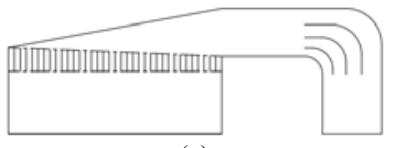

(a)
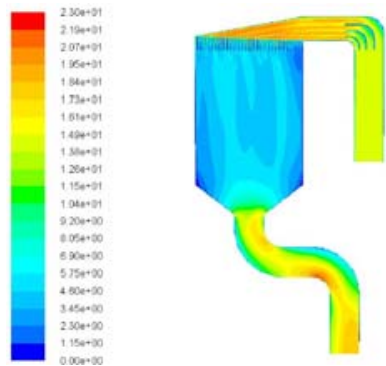

(b)

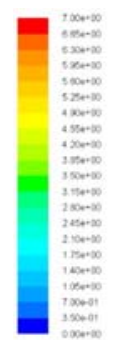

(c)

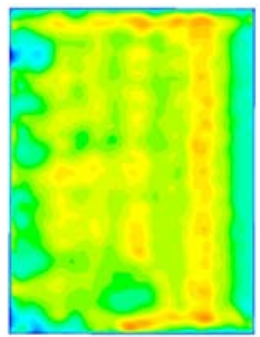

FIGURE VIII. THE DESIGN METHOD OF PLAN D (A) AND ITS VELOCITY CONTOURS OF AXIAL SYMMETRY PLANE B) AND CATALYST INLET SECTION (C)

\section{REFERENCE}

[1] Du YunGui, Yu Yu, Wu QiRong, Deng JiaJia. The Flow Modeling and Experiment Simulation of the DeNOx System[J]. Environmental Engineering, 2009(s1):255-257.

[2] Ma DongZhu, Zhang Ling, Li ShuShan, Yin Di. Application and Development of SCR Denitration Technology in Coal-fired Power Plant[J]. Coal Technology, 2011, 30(3):5-7.

[3] Xu YuanYuan, Zhang Yan, Wang JingCheng, Yuan JingQi. Application of CFD in the optimal design of a SCR-DeNO $\mathrm{x}$, system for a $300 \mathrm{MW}$ coal-fired power plant[J]. Computers \& Chemical Engineering, 2013, 49:50-60.

[4] Zhao DaZhou, Wang ChuanQi, Si FengQi, Xu ZhiGao. Influence of inlet velocity field and component concentration distribution on de- $\mathrm{NO}_{\mathrm{x}}$ efficiency of SCR denitration systems[J]. Thermal Power Generation, 2016, 45(2):29-33.

[5] Zhu TianYu, Li DeBo, Fang QingYan, Chen Gang. Flow Field Optimization for SCR System of Coal-fired Power Plants[J]. Chinese Journal of Power Engineering, 2015, 35(6):481-488.

[6] Cheng ZhiHai, Zhai YongQiang, Yu Jiang. Flow Field and Ammonia Spraying Optimization of SCR DeNOx System with Variable Cross Section in 600MW Coal-fired Unit[J]. Environmental Engineering, 2017, 35(10):95-99.

[7] Liu YingJin, Li JieYi, Li Bing, Wang YiBin, Liu Xing, Zhen ZhiGuang, et al. Optimization and Efficiency Improvement of SCR Flow Field of 330MW Coal-fired Unit[J]. Tecnology, 2017(6):67-71.

[8] Li Tan, Jin ShiPing, Huang SuYi, Liu Wei. Evaluation Indices of Flow Velocity Distribution Uniformity: Comparison Application[J]. Thermal Power Generation, 2013, 42(11):60-63.

[9] Wang DengHui, Hui ShiEn, Liu ChangChun, Shang Tong, Zou Hao, Zhang Geng. Effect of the Shape of SCR Reaction Tower and the Internal Guiding Plates on the Airflow Uniformity[J]. Energy Procedia, 2014, 61(ECCE):398-401.

[10] Xu Yan, Li WenYan. Structure Design of Baffle Plates in SCR Denitrification Reactor[J]. Thermal Power Generation, 2008, 37(10):4952. 